\title{
Global mapping for the epidemiology of paediatric spinal cord damage: towards a living data repository
}

\author{
Peter Wayne New $\mathbb{C}^{1,2,3} \cdot$ Bonsan Bonne Lee ${ }^{4,5} \cdot$ Raymond Cripps $^{6} \cdot$ Lawrence C. Vogel $\mathbb{C}^{7}$. \\ Adam Scheinberg $\mathbb{1 0}^{8,9,10} \cdot$ Mary-Clare Waugh $\mathbb{1}^{11,12}$
}

Received: 24 May 2018 / Revised: 23 September 2018 / Accepted: 7 October 2018 / Published online: 14 December 2018

(c) International Spinal Cord Society 2018

\begin{abstract}
Study design Literature review.

Objectives Globally map key paediatric spinal cord damage epidemiological measures and provide a framework for an ongoing repository of data.

Setting Worldwide, initiative of ISCoS Prevention Committee.

Methods Literature search of Medline (1946-March 2017) and Embase (1974-March 2017). Relevant articles in any language regarding children with spinal cord damage included. Stratification of data quality into Green/Yellow /Red 'zones' facilitated comparison between countries.

Results A total of 862 abstracts were reviewed and data from 25 articles were included from 14 countries in 6 of the 21 Global Regions. Fourteen studies involved paediatric traumatic spinal cord injury (SCI) and seven were regarding nontraumatic spinal cord dysfunction (SCDys). An additional four articles provided both paediatric SCI and SCDys data. The median SCI incidence rates in Global Regions were: Asia, East 5.4/million population/year; Australasia 9.9/million population/year; Western Europe 3.3/million population/year and North America, High Income 13.2 million population/ year. The median SCDys incidence rates in Global Regions were: Australasia 6.5/million population/year; Western Europe 6.2/million population/year and North America, High Income 2.1/million population/year. SCI was mostly due to land transport (46-74\%), falls (12-35\%) and sport/recreation (10-25\%) and SCDys was mostly caused by tumours (30-63\%) and inflammatory/autoimmune causes $(28-35 \%)$.

Conclusions There is a scarcity of quality epidemiology studies of paediatric SCD regarding incidence, prevalence, aetiology and survival. Recent ISCoS frameworks provide guidance for researchers to use established classification of SCDys and age group cut-off levels in future studies, thereby improving the ability to compare and combine data.
\end{abstract}

\section{Introduction}

Compared with adults, there is very little epidemiological information available about paediatric spinal cord damage (SCD). It is well documented that paediatric SCD arises from diverse causes and includes traumatic spinal cord injury (SCI) and non-traumatic aetiologies, which, in the absence of an internationally accepted term [1], we

Electronic supplementary material The online version of this article (https://doi.org/10.1038/s41393-018-0209-5) contains supplementary material, which is available to authorised users.

Peter Wayne New

p.new@alfred.org.au

Extended author information available on the last page of the article will refer to here as spinal cord dysfunction (SCDys). Prior global mapping publications use the term nontraumatic SCI, but SCDys is believed to be a more appropriate term for this group of patients. Studies of the incidence, aetiology, prevalence and survival of children with SCD assists in planning health care and service delivery and facilitates the development of preventive strategies.

The International Spinal Cord Society (ISCoS) Prevention Committee has presented epidemiological information on SCI [2] and SCDys [3] in adults. These enhanced mapping techniques are just as relevant for paediatric SCD in documenting incidence, prevalence aetiology and survival. The global maps, which highlight the limitations of existing literature discussed below, can be used to provide direction for improving future epidemiological studies if researchers 
following our recommendations. In addition, they can be used to focus the efforts of national and regional collaborations regarding prevention of paediatric SCD. This paper is part of a worldwide spinal cord injury mapping project undertaken by the ISCoS Prevention Committee which aims to:

1. Disseminate paediatric SCD data through the Global Map for Spinal Cord Injury Epidemiology (http:// iscos.org.uk/page.php?content $=57$ )

2. Create partnerships within ISCoS to facilitate the development of strategies for primary and secondary prevention of paediatric SCD.

3. As with the adult maps, establish an electronic data repository accessible through the ISCoS website to enable simple conversion of key demographic and epidemiological information into a graphical, easily interpretable and updatable map of global trends in paediatric SCD.

It is intended that this work continue as an ongoing project which will be open to contributions of published and unpublished data to assist the ISCoS Prevention Committee and other organisations in the development of paediatric SCD prevention programs.

The aim of this project is to report the results of a comprehensive literature search and to compile global maps summarising the epidemiology of paediatric SCD, with a focus on the incidence, prevalence, aetiology, and survival, as well as the level of SCD. The results for SCI and SCDys will be reported separately.

\section{Methods}

Literature searches of Medline (1946-March 2017, inclusive) and Embase (1974-March 2017, inclusive) were performed without date or language restrictions for publications or reports pertaining to paediatric SCD from any cause, focusing on the aetiology, incidence, prevalence and mortality. See Appendix 1 in the supplementary information for the search strategy.

Following completion of the literature search, we became aware of an additional publication of relevance that was published regarding data from Ireland [4]. Given the dearth of literature on the subject of this review, it was decided that this paper would also be included.

Publications and reports in any language were reviewed. Translation was arranged where appropriate to determine eligibility and for extraction of relevant information. It was decided that reports dealing specifically with spina-bifida would be excluded because of the existence of registries in numerous countries dealing with this condition.
We provisionally used an age cut-off for inclusion of less than 15 years. This was to avoid overlap between the paediatric global maps and the adult global maps published previously in 2014 that included people aged 15 years and above [2, 3]. This approach is also consistent with the recently revised version of the International SCI core dataset [5]. In some instances, there were references that had age cut-offs less than 19 years, but because of the paucity of data, these were included and were notated in the tables as having a different age cut-off.

Former World Health Organisation global regions (referred to in this paper as Global Regions) were utilised to allow epidemiologically similar zones to be compared [6]. Regional data was divided into four broad zones (Box 1) and the most representative studies selected.

Maps using SCI and SCDys incidence and aetiology data were developed for Global Regions and countries within these regions using mapping and graphical techniques developed by Myriad Editions http://www.myriadeditions. com/infographics/). Median/ average values of country data were used for mapping Global Regions and countries within these regions. ISCoS has developed the International Nontraumatic Spinal Cord Injury Data Set, which included a detailed classification of SCDys [7]. It is expected that when standardised categories of SCDys are used in future outcome studies, the reporting of SCDys data from a global perspective will improve. This project used the ISCoS classification of SCDys aetiologies, with some categories collapsed or omitted, as recommended, because of the absence or rarity of cases reported. Level of SCD, when reported, was described as either paraplegic or tetraplegic.

Sources of data used were primarily from peer-reviewed journal publications and governmental reports. Studies were prospective and retrospective and used data from spinal registries, population registries, hospital data (admission and discharge data) and health surveys. The quality of the information in the various publications was appraised using a grading system to guide readers in interpreting the results and conclusions reached. Each paper's quality of evidence is graded using the following annotation: High $(\mathrm{H})$, Moderate (M), and Low (L) and is summarised in Box 2.

\section{Results}

There were 25 peer-reviewed articles that met the inclusion criteria and were included in this study. Fourteen of these were studies of traumatic SCI [8-21], seven were of nontraumatic SCDys [22-28], and an additional four articles provided both paediatric SCI and SCDys data [4, 29-31]. Details of the reference data sources and their quality ranking of data (5 Green zone $(\mathrm{H}), 2$ Yellow zone $(\mathrm{H}), 11$ 
Yellow zone (M), 3 Orange zone (M) and 2 Red zone (L)) are summarised in Tables 1 and 2.

Globally, paediatric data from 6 Regions and 12 countries within those Regions were identified and are summarised in Tables 3 and 4 and Figs. 1-4. Due to the paucity of paediatric SCD data, interpretation of Global Region and country data within these Regions should be made with caution. There were many publications excluded because they were single-unit case-series, and not population-based (Red Zone), and there was better quality data available in the region. Unfortunately, some regions only have red zone data, and these have been indicated in the results below and on the maps.

The median SCI incidence rates in Global Regions were: Asia, East 5.4/million population/year; Australasia, 9.9/million population/year; Western Europe 3.3/million population/year and North America, High Income 13.2 million population/year. The median SCDys incidence rates in Global Regions were: Australasia 6.5/million population/ year; Western Europe 6.2/million population/year and North America, High income 2.1/million population/year.

The SCI prevalence rates were: Australia, average estimate of 51.5 cases per million population in 2011; Canada, 10.1 cases per million population in 2010; and Ireland, 12.1 cases per million population in 2015. For SCDys the prevalence rates were: Australia, 6 per million population in 2010; Canada, 2.5 cases per million population in 2010; and Ireland, 19.6 cases per million population in 2015 .

There were very few studies on survival following SCD, with most having very small sample sizes. The best study of survival following SCI was from the USA, which found that children who were less than 16 years of age at time of SCI had a $31 \%$ increase in the annual odds of dying compared with persons injured at older ages. The increased risk did not vary significantly by current age, sex, race, injury severity, or decade of injury. The only studies of survival in children with SCDys involved tumours, and had small sample sizes.

SCI was mostly due to land transport (46-74\%), falls (12-35\%) and sport/recreation (10-25\%) and SCDys was mostly caused by tumours (30-63\%) and inflammatory/ autoimmune causes (28-35\%). There were few studies where the level of SCD was reported, with most SCI and SCDys reported to result in paraplegia.

\section{Paediatric spinal cord damage}

\section{Asia Pacific, High income}

Republic of Korea: Data were obtained regarding SCI from the Department of Physical Medicine and Rehabilitation, for the period January 1995 to July 2006 and the catchment
Box 1 Enhanced zones of information*

1.Green Zone: Global region or country with a prospective spinal cord injury register (PSCIR) or population health registry (including population registries linked or able to be linked to health and/or mortality data)

2.Yellow Zone: Partial coverage by PSCIR or population health registry

3.Orange Zone: Extrapolated data (not directly collected through a formal registry but derived from multiple sources with documented assumptions). Dependent on the assumptions and methods used, caution needs to be made in interpreting this data.

Red Zone: No PSCIR or population health registry data available and no extrapolated data available. This information is considered to be of insufficient quality to make between global region or country comparisons on the basis of incidence.

*Incidence data are rated on the SCI and SCDys global maps using these colours

Box 2 Individual quality ranking of data

High $(\mathrm{H})$ : Green/Yellow Zone data AND nationally representative data;

Moderate (M): Green/Yellow Zone data AND NOT nationally representative data or Orange Zone data (national data through adjusted regression techniques) or Red Zone data AND nationally representative data;

Low (L): Other Red Zone data;

NA: Not applicable due to patient population

The initial grading was performed by $\mathrm{BBL}$ and $\mathrm{RC}$, with subsequent rounds reviewed by clinicians (PWN, BBL, LV, $\mathrm{MCW}, \mathrm{AS}$ ) who independently reviewed titles and abstracts of potentially relevant papers identified in the literature search. The papers identified as potentially relevant were then screened by (BBL and RC) to confirm eligibility. Any conflicting assessments were adjudicated by PWN, who made the final determination.

A flow chart giving the results of the search strategy is shown in Appendix 2, available in the supplementary information.

area of Asan Medical Center, Seoul for children were aged $0-12$ years [31]. Patients $13-18$ years of age $(n=22)$ were excluded from this study as the majority of cases were greater than 15 years. In total, 26 cases of paediatric SCD were identified, of which 9 (34.6\%) were SCI and 17 (65.4\%) SCDys. Quality ranking of data is Low and Red Zone data (See Boxes 1 and 2).

In the SCI cohort, the most common cause of SCI was motor vehicle accidents, which accounted for $89 \%(n=8)$. The other cause of injury was due to a fall in a child over 4 years of age.

For the SCDys cohort, the most common causes of SCDys were tumour and inflammation, which accounted for $59 \%(n=10)$ of the cases. The remaining causes of SCDys were due to congenital and unspecified aetiology $(41 \%, n=7)$. 


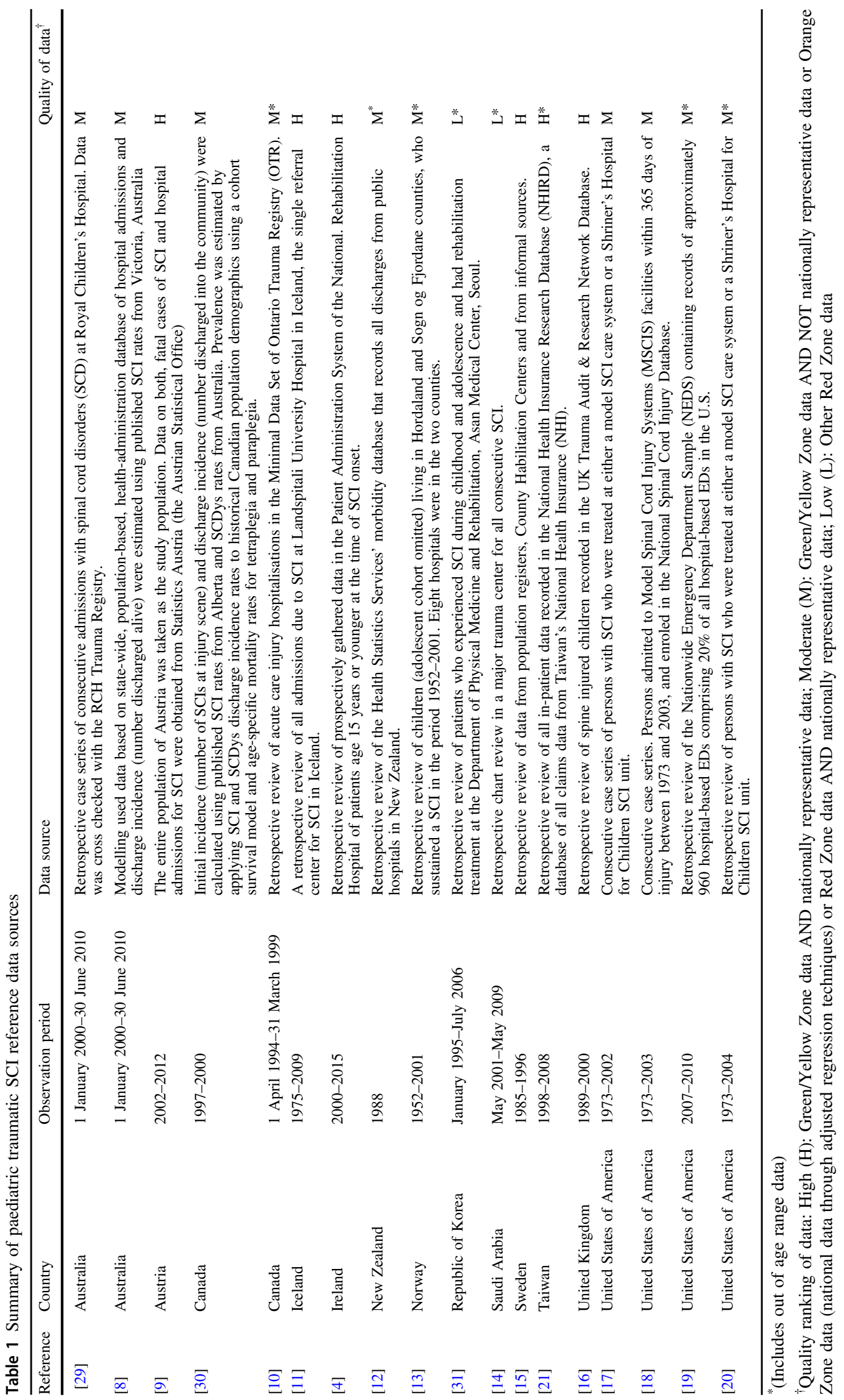




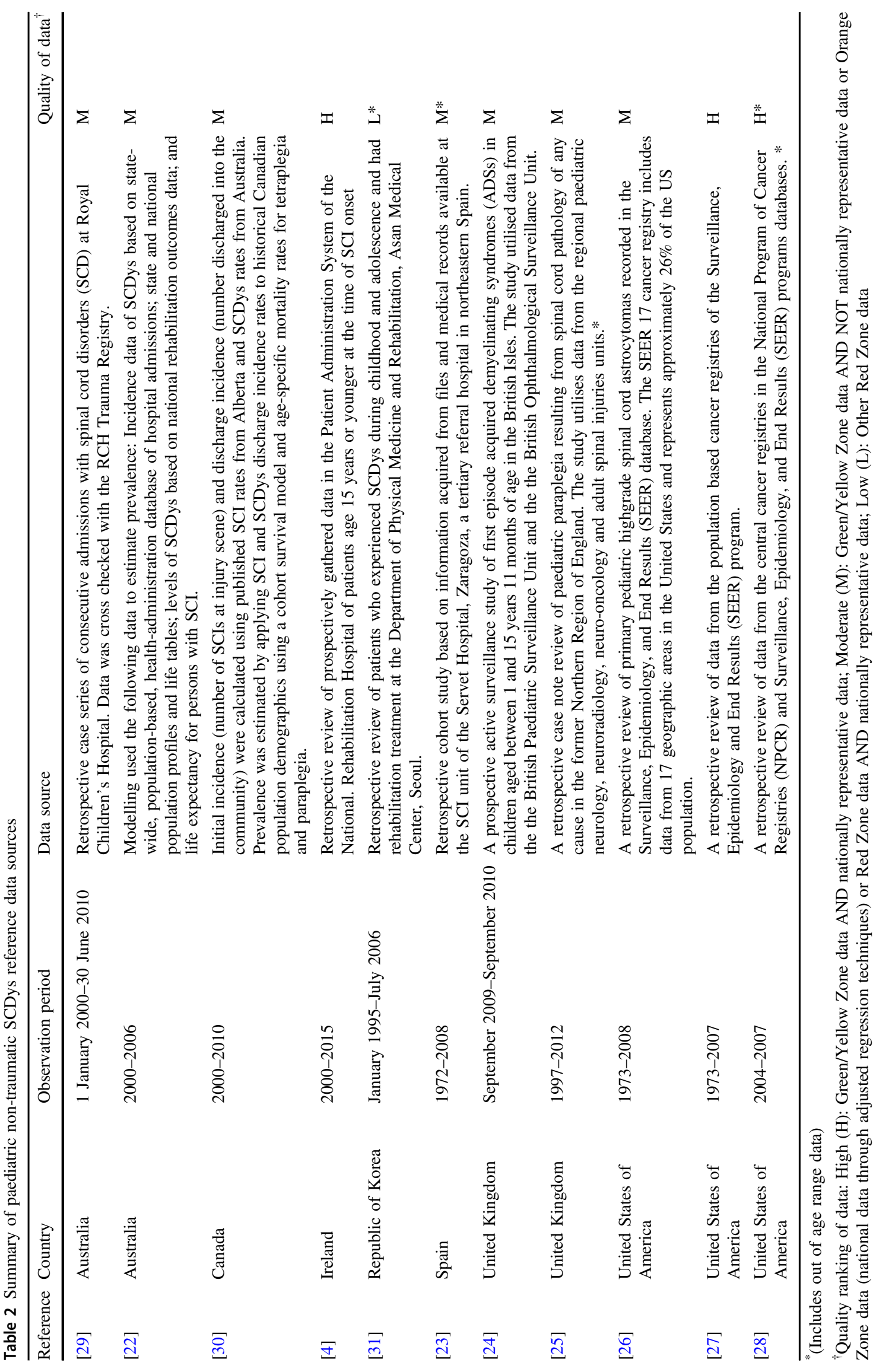




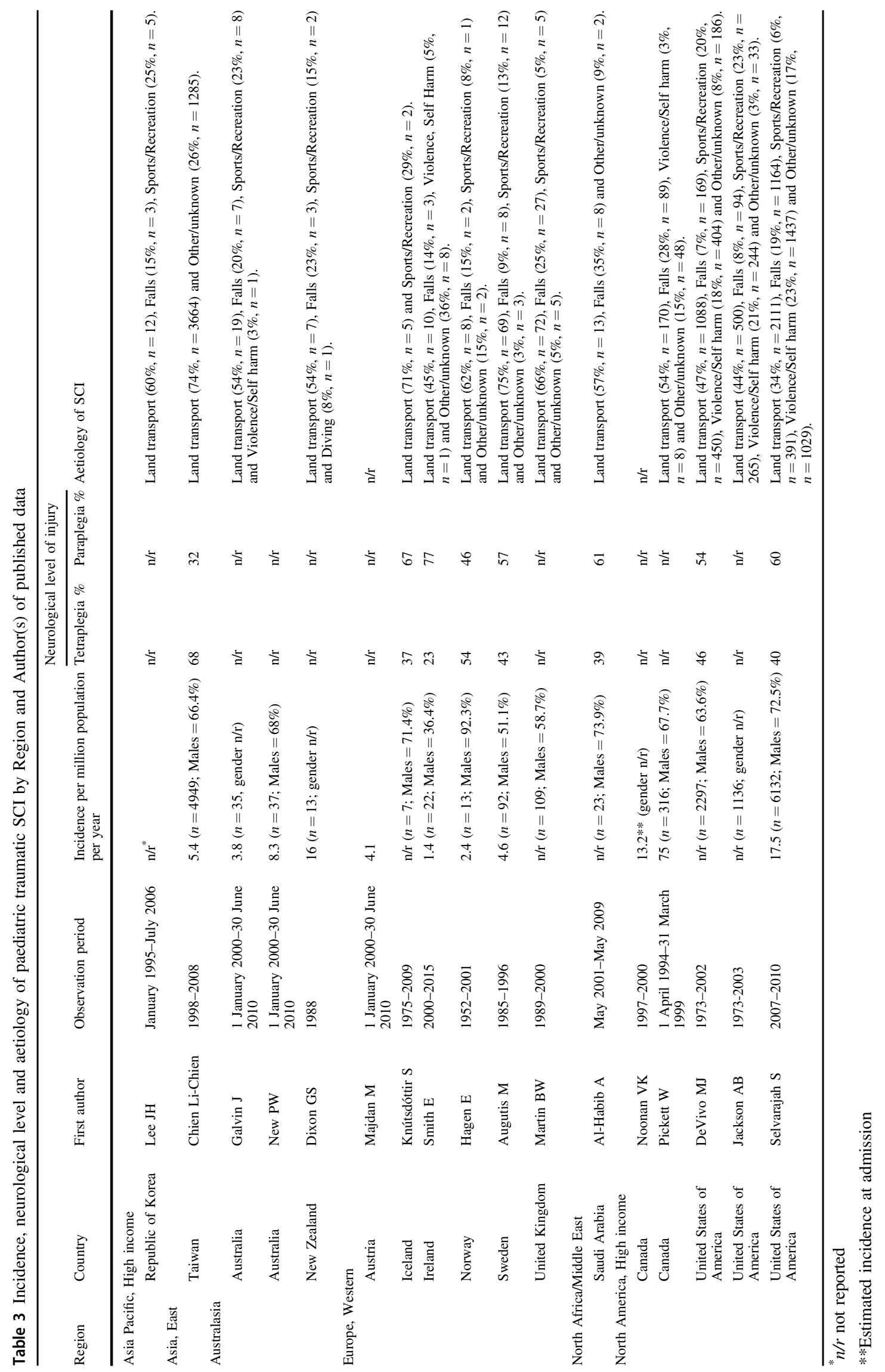




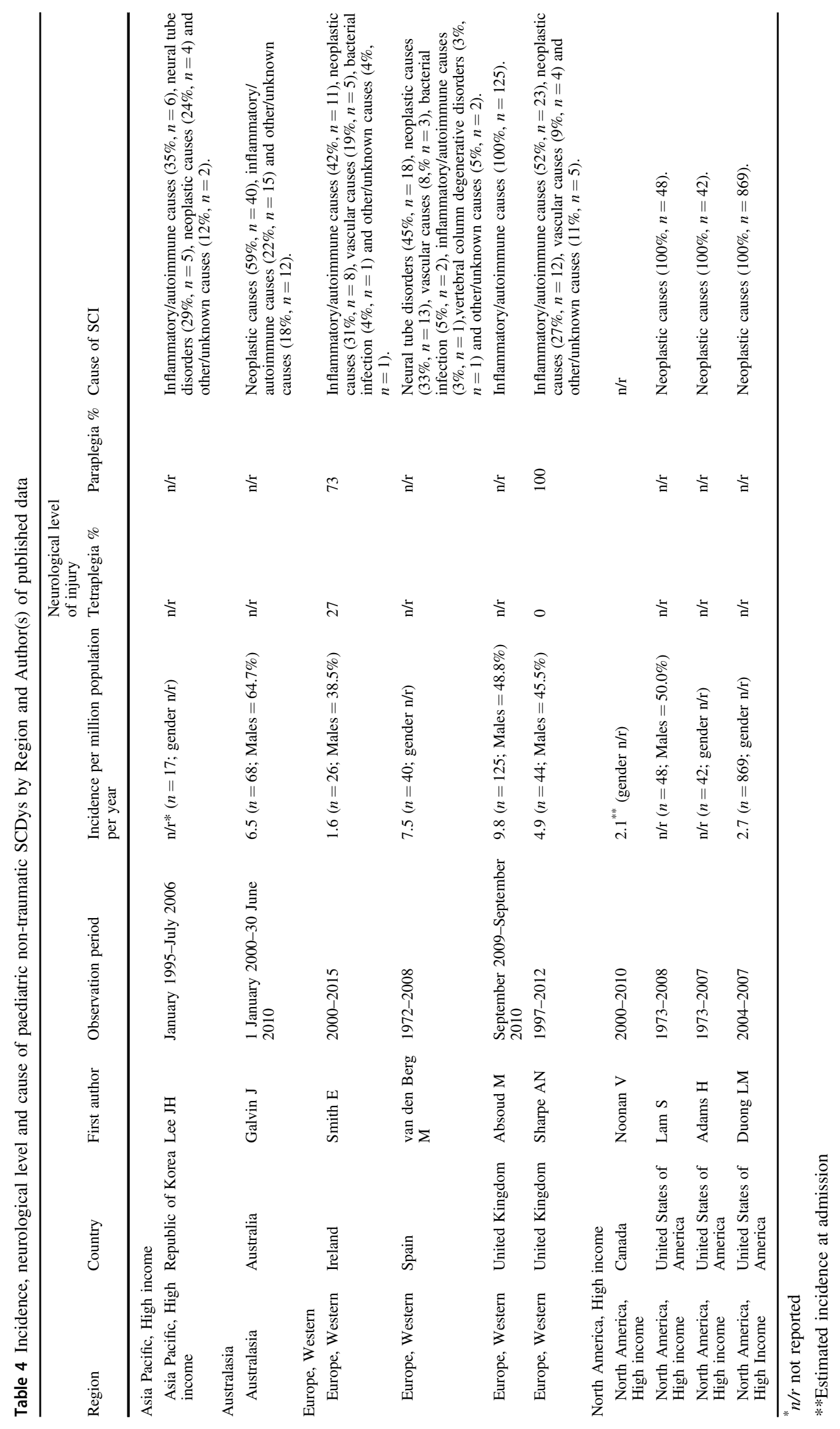




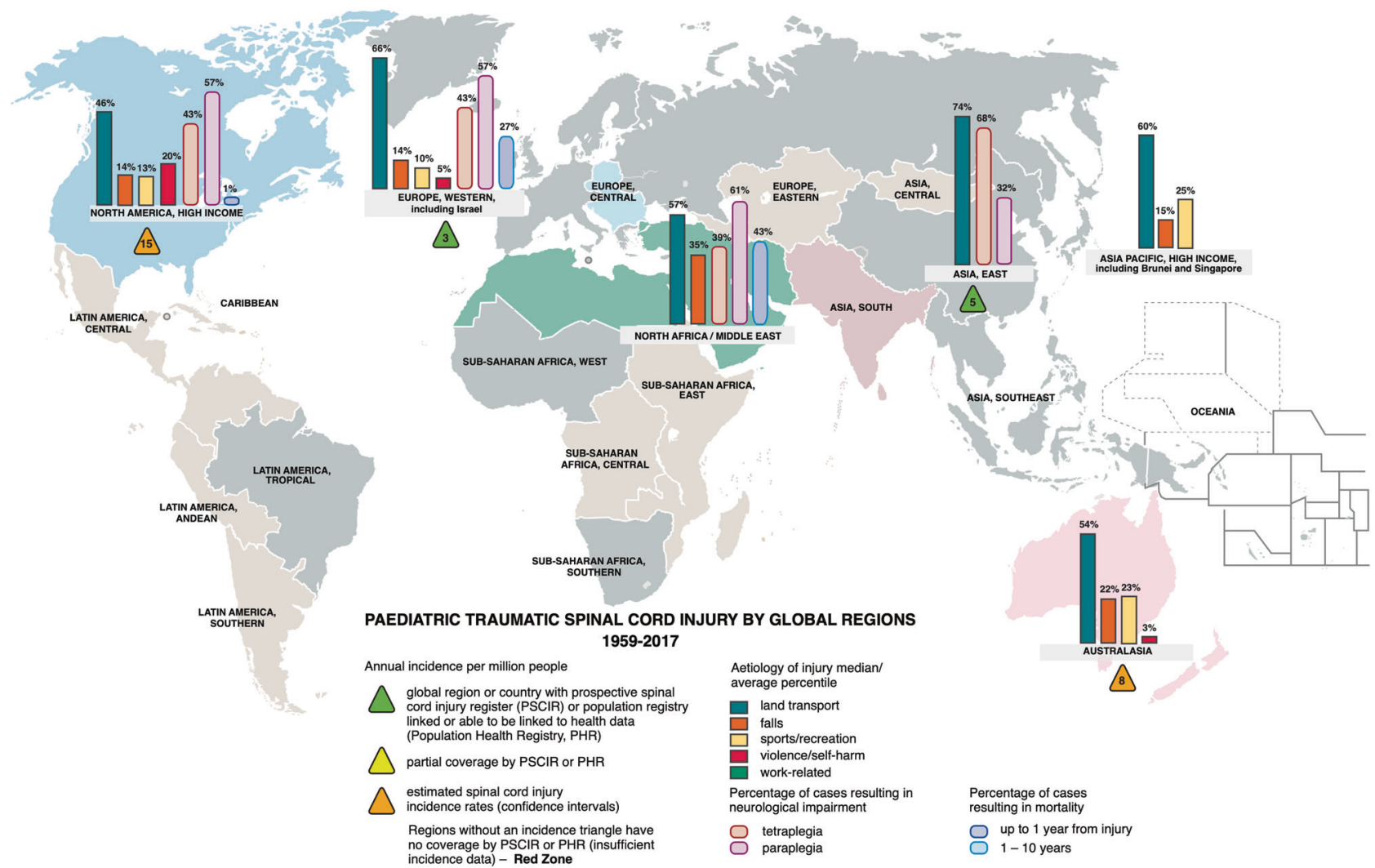

Fig. 1 Paediatric traumatic SCI by global regions

\section{Asia East}

Taiwan: Data were obtained regarding SCI from the National Health Insurance Research Database, for the whole country, for the period 1998-2008, for children aged 0-18 years [21]. Quality ranking of data is High and Green Zone data. In total, 4949 cases of paediatric SCI were identified. This equates to an incidence rate for SCI of 59.9 cases per million population per year. Quality ranking of data is High and Green Zone data

Of the 4949 SCI cases, 66.4\% $(n=3284 / 4949)$ were male. In $68.5 \%(n=3351)$ of the SCI cases, the level of injury involved the cervical spinal cord and were predominately male. The most common cause of SCI was motor vehicle accidents, which accounted for $74 \% \quad(n=$ $3664)$ of the cases. No data was reported for SCDys.

\section{Australasia}

Australia: Three studies were located from Australia.

Data were obtained regarding both SCI and SCDys from the Royal Children's Hospital, Melbourne, for the period 2000-2010 and the catchment area of the State of Victoria for children were aged 0-15 years [29]. In total, 103 cases of paediatric SCD were identified, of which 35 (34\%) were
SCI and 68 (66\%) SCDys. This equates to an incidence rate for Victoria of 3.8 SCI cases and 6.5 SCDys cases per million population per year. Quality ranking of data is Moderate and Yellow Zone data.

Of 35 SCI cases, 69\% $(n=24 / 35)$ were male. In this cohort, the most common cause of SCI was motor vehicle accidents, which accounted for $54 \%(n=19)$. Other causes included: $20 \%(n=7)$ from high falls, $23 \%(n=8)$ from sporting activities and $3 \%(n=1)$ from assault.

Of 68 SCDys cases, 65\% $(n=44 / 68)$ were male. The most common cause of SCDys was tumour, which accounted for $63 \% \%(n=43)$. Other causes included $29 \%$ $(n=20)$ from transverse myelitis and $7 \%(n=5)$ from other diseases of the spinal cord.

Another study, using computer modelling and two population-based databases of hospital separations of patients with SCI in Victoria between 2000 and 2010 were used to estimate SCI incidence in Australia [8]. The paediatric cohort in this study was aged 0-15 years and were discharged alive. Quality ranking of data is Moderate and Orange Zone data.

Of the paediatric cases, $16(75 \%, n=12$ male $)$ were used to derive the lower estimate incidence rate of $3.6 \mathrm{SCI}$ per million population per year and $58(66 \%, n=38$ male) to derive the upper estimate incidence rate of 12.9 SCI per 


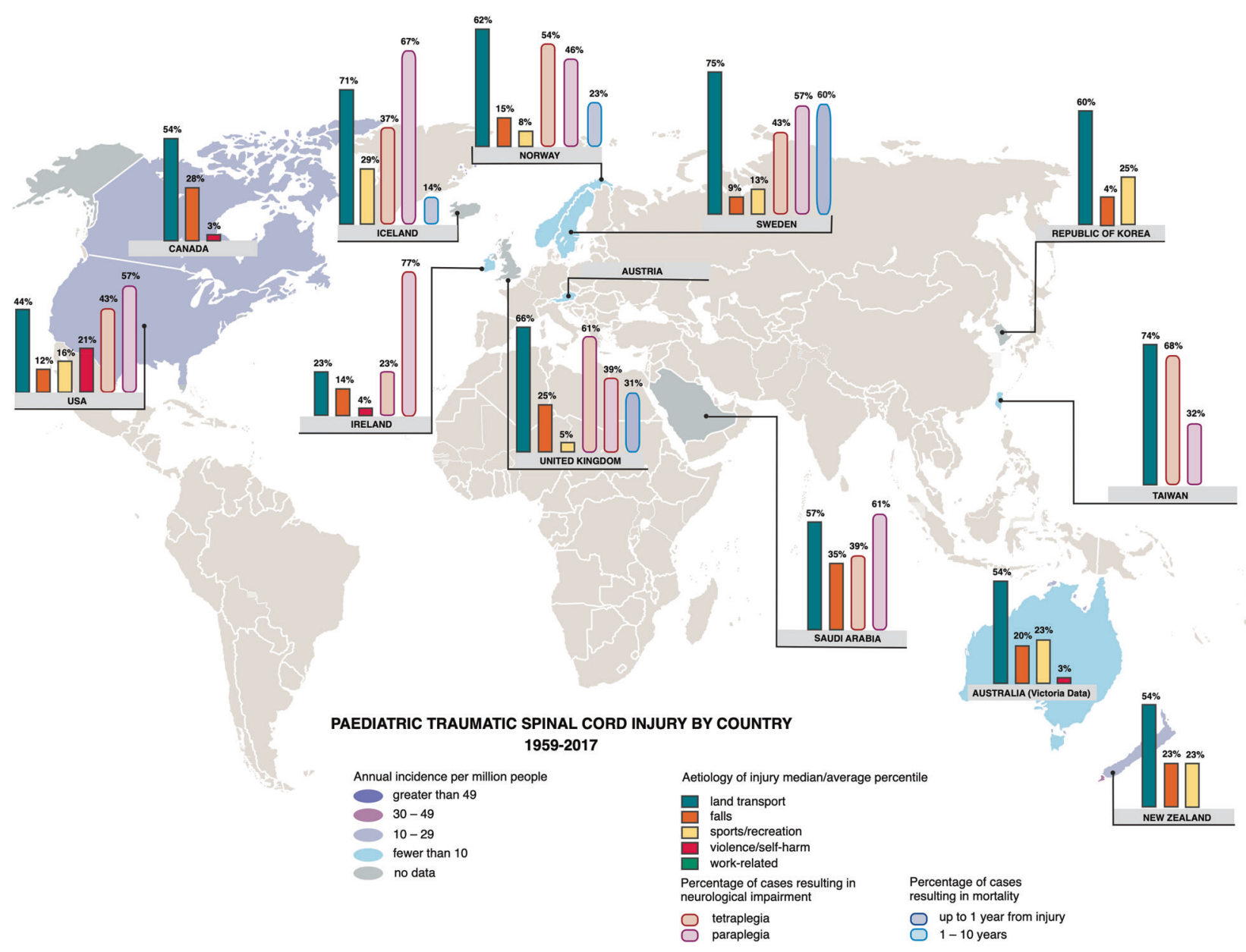

Fig. 2 Paediatric traumatic SCI by country

million population per year. These lower and upper SCI incidence estimates average at $8.3 \mathrm{SCI}$ per million population. per year. The lower estimate of the prevalence of SCI in 2011 was 17 per million population and the upper estimate was 86 per million population, giving an average estimated prevalence of 51.5 per million population.

Another study, also involving computer modelling, estimated the prevalence of SCDys in 2010 [22]. The model used results from a previous study utilising a State-wide database of hospital separations between 2000 and 2006 and ICD-10 codes to identify SCDys patients in Victoria, Australia. The paediatric cohort in this study was aged $0-15$ years and were discharged alive. Quality ranking of data is Moderate and Orange Zone data.

The estimated prevalence of SCDys in 2010 was 6 per million population.

New Zealand: National data on SCI were obtained from the New Zealand Health Statistics Services for the year 1988 [12]. In total, 13 cases of paediatric SCI aged $0-14$ years were identified. This equates to an incidence rate for SCI of 16 SCI cases per million population per year. No data was reported on SCDys.
Quality ranking of data is Moderate and Yellow Zone data.

In this cohort, the most common cause of SCI was land transport accidents, which accounted for 54\% $(n=7)$, with children injured as passengers, pedal cyclists and pedestrians. Other causes included: high falls $(23 \%$, $n=3)$, sports-related injury $(15 \%, n=2)$ and diving $(8 \%$, $n=1)$.

\section{Europe, Western}

Austria: Administrative data were obtained regarding SCI from the Austrian Statistical Office for the period 2002-2012 and the catchment area of the entire population of Austria for children were aged $0-14$ years [9]. This equates to an incidence rate for SCI of 4.1 per million population per year. No data was reported on SCDys. Quality ranking of data is High and Green Zone data.

Iceland: Data were obtained regarding SCI from Landspitali University Hospital in Iceland, the single referral center for SCI in Iceland for the period 1975-2009 and the catchment area of Iceland for children aged 0-14 years [11]. 


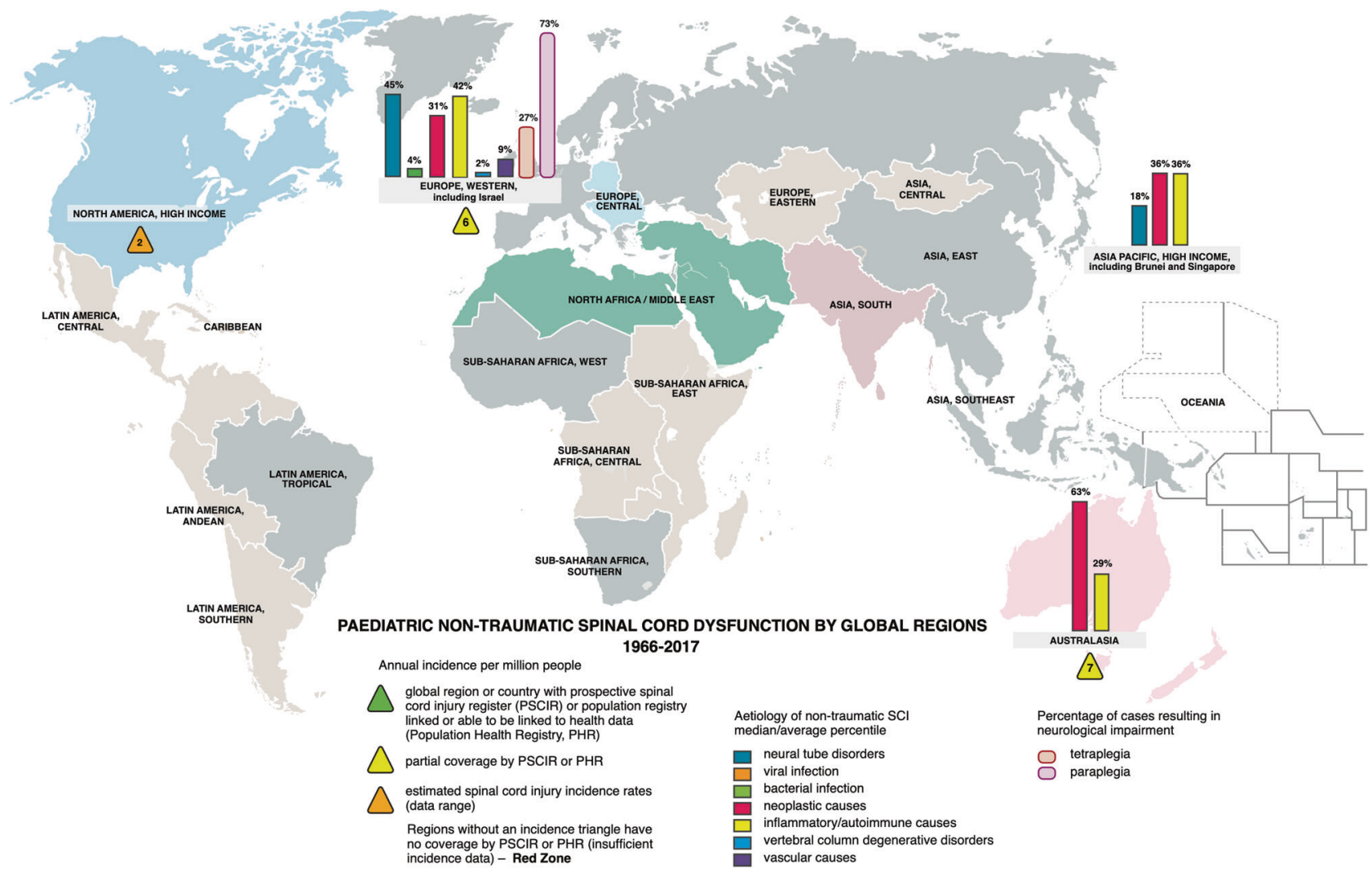

Fig. 3 Paediatric non-traumatic SCDys by global regions

In total, 7 cases of paediatric SCI were identified. No data was reported on SCDys. Quality ranking of data is High and Green Zone data.

Of 7 SCI cases, $71 \%(n=5 / 7)$ were male. In $37 \%(n=3)$, the level of injury involved the cervical spinal cord. In this cohort, the most common cause of SCI was land transport, which accounted for $71 \%(n=5)$ land transport. With the remainder due to porting/recreational activities $(29 \%, n=2)$. The 12-month mortality for SCI was $14 \%(n=1)$.

Ireland: Data were obtained from the patient admission system of the National Rehabilitation Hospital for the period 2000-2015 and the catchment area of Ireland for children were aged $0-15$ years [4]. In total, 48 cases of paediatric SCD were identified, of which 22 (45.8\%) were SCI and 26 (54.2\%) SCDys. This equates to an incidence rate of 1.4 SCI cases and 1.6 SCDys cases per million population per year. The prevalence at 2015 was 12.1 SCI cases and 19.6 SCDys cases per million population. Quality ranking of data is High and Green Zone data.

Of 22 SCI cases, $36.4 \%(n=8 / 22)$ were male. In $22.7 \%$ $(n=5)$, the level of injury involved the cervical spinal cord. In this cohort, the most common cause of SCI was land transport, which accounted for $45.5 \%(n=10)$. Other causes included: surgical complications, accounting for $36.4 \%$ $(n=8)$, falls $(13.6 \%, n=3)$ and violence $(4.5 \%, n=1)$.
Of 26 SCDys cases, $38.5 \%(n=10 / 26)$ were male. In $28 \%(n=7)$, the level of injury involved the cervical spinal cord. In this cohort, the most common cause of SCDys was transverse myelitis, which accounted for 42.3\% ( $n=11)$. Other causes included: tumour, accounting for $32 \%(n=8)$, vascular $20 \%(n=5)$, infection $(4 \%$, $n=1)$ and other $(4 \%, n=1)$.

Norway: Data were obtained regarding SCI from 8 SCI hospitals for the period 1952-2001 and the catchment area of the Hordaland and Sogn og Fjordane counties for children were aged $0-14$ years [13]. In total, 13 cases of paediatric SCI were identified. This equates to an incidence rate for SCI of 2.4 cases per million population per year. No data was reported on SCDys. Quality ranking of data is Moderate and Yellow Zone data.

Of 13 SCI cases, $92 \%(n=12)$ were male. In $54 \%(n=$ 7 ), the level of injury involved the cervical spinal cord and $77 \%(n=10)$ of the SCI cases were complete. In this cohort, the most common cause of SCI was land transport, which accounted for $62 \%(n=8$; pedestrians $[n=4]$, motor vehicles $[n=3]$ and bicycles $[n=1])$. Other causes included: $15 \%(n=2)$ from falls, $8 \%(n=1)$ from sporting activities and $15 \%(n=2)$ other causes. The 12-month mortality for SCI in this population was $23 \%(n=3)$, all had injuries to the cervical spinal cord. 


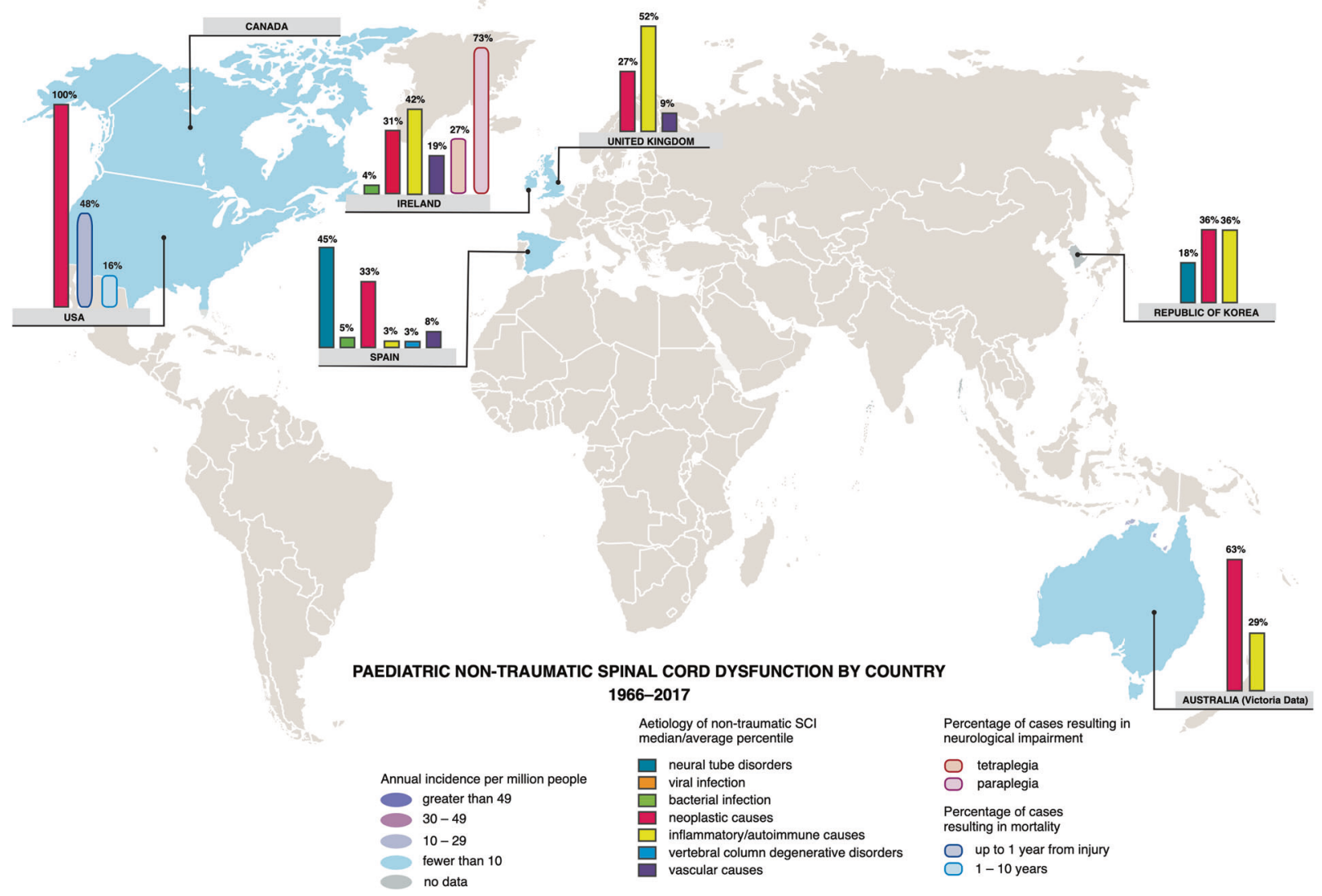

Fig. 4 Paediatric non-traumatic SCDys by country

Spain: Data for this study was obtained from a retrospective cohort study of inpatients and outpatients from the Servet Hospital in Zaragoza, Spain, between January 1972 and December 2008. The SCI unit in this hospital serves the catchment area of the autonomous region of Aragón [23]. Figures 1 and 2 in the reference paper were used to estimate the number of paediatric cases and aetiology in the cohort 0-19 years. The number of SCDys cases was estimated to be about 40 and the estimated incidence rate for this paediatric group was 7.5 per million population per year. Quality ranking of data is Moderate and Yellow Zone data.

The number of female cases was slightly higher than male cases, but no exact numbers were available. Forty-five per cent $(n=18)$ had neural tube disorders, 5\% $(n=2)$ bacterial infection, 33\% $(n=13)$ neoplastic causes, $3 \%(n$ $=1)$ inflammatory causes, $3 \%(n=1)$ vertebral column degenerative disorders, $8 \%(n=3)$ vascular causes and other/unknown $5 \%(n=2)$.

Sweden: Data were obtained regarding SCI from population registers and other Swedish data sources for the period 1985-1996 for children were aged 0-15 years [15]. In total, 92 cases of paediatric SCI were identified. This equates to an incidence rate for SCI of 4.6 cases per million population per year. No data was reported on SCDys. Quality ranking of data is High and Green Zone data.
Of the 92 total SCI cases, $51 \%(n=47)$ were male. In $43 \%(n=40)$ the level of injury involved the cervical spinal cord with cervical lesions among the survivors $(n=37)$ as common as thoracic ones $(n=16)$. Almost half of the survivors $(n=17)$ had a complete SCI (ASIA A). In this cohort of 92 cases, the most common cause of SCI was land transport, which accounted for $75 \%(n=69$; of these, $43 \%$ passengers, $26 \%$ pedestrians, $16 \%$ bicyclists and $14 \%$ Moped riders). Other causes included falls (primarily high falls; $9 \%, \mathrm{n}=8)$ and sports-related accidents $(13 \%, n=12)$. The 12-month mortality for SCI was $60 \%(n=55)$.

United Kingdom: Three studies were located from the UK.

Data were obtained regarding SCI from the UK Trauma Audit \& Research database for the period 1989-2000 and the catchment areas of England and Wales for children were aged $0-15$ years [16]. In total, 109 cases of paediatric SCI were identified. No data was reported on SCDys. Quality ranking of data is High and Green Zone data

Of 109 SCI cases, $59 \%(n=64)$ were male. In $66 \%(n=$ 72), the level of injury involved the cervical spinal cord. In this cohort of 109 SCI cases, the most common cause of SCI was land transport, which accounted for $66 \%(n=72)$. Other causes included: falls $(25 \%, n=27)$ and sport activities $(5 \%$, $\mathrm{n}=5)$. Hospital mortality for SCI was $31 \%(n=34)$. 
Data were obtained regarding SCDys causing acute paraplegia from multiple neurological treatment centres' databases for the period 1997 and March 2012 in the Northern region of England for children were aged 0-15 years [25]. In total, 44 cases of paediatric SCDys causing paraplegia were identified. This equates to an incidence rate for SCDys of 4.9 cases per million population per year. No data was reported on SCI. Quality ranking of data is Moderate and Yellow Zone data.

Of 44 cases, $45.5 \% \quad(n=20 / 44)$ were male. In this cohort, the most common cause of SCDys was inflammation, which accounted for $52 \%(n=23)$. Other causes included: neoplasms $(27 \%, n=12)$, vascular $(9 \%, n=4)$ and other/unknown causes $(11 \%, n=5)$.

Data were obtained regarding SCDys due to acquired demyelinating syndromes (initial onset) by active surveillance of all paediatricians, and ophthalmologists for the period 2009-2010 and the catchment area of British Isles for children were aged 1-15 years [24]. In total, 125 cases of paediatric SCDys due to acquired demyelinating syndromes were identified. This equates to an incidence rate for SCDys due to acquired demyelinating syndromes of 9.8 cases per million population per year. No data was reported on other causes of SCDys or SCI. Quality ranking of data is Moderate and Yellow Zone data.

Of 125 SCDys cases due to acquired demyelinating syndromes, $48.8 \%(n=61)$ were male. Two percent $(n=3)$ died within the first year.

\section{North Africa/Middle East}

Saudi Arabia: Data were obtained for SCI, retrospectively collected from the trauma registry database at King Abdulaziz Medical City for the period 2001-2009 and the catchment area of Riyadh for children were aged 0-19 years [14]. In total, 23 cases of paediatric SCI were identified. No data was reported on SCDys. Quality ranking of data is Low and Red Zone data.

Of the 23 SCI cases, $74 \%(n=17)$ were male. In $39 \%$ $(n=7)$, the level of injury involved the cervical spinal cord. In this cohort, the most common cause of SCI was land transport, which accounted for $57 \%(n=13)$. Most of these cases were young children $<12$ years of age injured as pedestrians and adolescent males injured as unrestrained drivers or passengers of motor vehicles). Other causes included: falls, accounting for $35 \%(n=8)$ and other/ unknown $(9 \%, n=2)$.

\section{North America, High income}

Canada: Two studies were located from Canada.

Data were obtained regarding SCI from the Minimal Data Set of the Ontario Trauma Registry hospital admissions for the period 1994/1995-1998/1999 and the catchment area of Ontario for children aged 0-19 years [10]. In total, 316 cases of paediatric SCI were identified. This equates to an estimated (based on Fig. 2 in reference paper) incidence rate for SCI of approximately 75 cases per million population per year. No data was reported on SCDys. Quality ranking of data is Moderate and Yellow Zone data.

Of 316 SCI cases, $67.7 \%(n=214)$ were male. In this cohort, the most common cause of SCI was land transport crash, which accounted for $54 \%(n=170)$. Other causes included: falls $(28 \%, n=89)$ violence/self harm $(3 \%, \mathrm{n}=8)$ and other/unknown $(15 \%, \mathrm{n}=48)$.

In another study, data were obtained regarding both SCI and SCDys using SCI published rates for Alberta and SCDys rates from Australia, data from the literature and computer modelling to derive estimates of SCI and SCDys incidence and prevalence for Canada in 2010 for children aged 0-14 years [30]. The estimated incidence rate for children discharged alive with SCI was 10.3 cases per million population per year and for SCDys it was 2.0 cases per million population per year. The estimated prevalence rates (Personal communication, Vanessa Noonan) were $10.1 /$ million population $(n=343)$ for SCI and $2.5 /$ million population $(n=86)$ for SCDys. Quality ranking of data is Moderate and Orange Zone data.

United States of America: Six studies were located from the USA, 3 each regarding SCI and SCDys.

Data for SCI were obtained from the USA Model Spinal Cord Injury Systems and Shriners Hospital for Children SCI unit for the period 1973-2002 and the catchment area of USA for children aged 0-15 years [17]. In total, 2297 cases of paediatric SCI were identified. Quality ranking of data is Moderate Yellow and Orange Zone data.

Of 2297 SCI cases, $63.6 \%(n=1,462)$ were male. In $46 \%$ $(n=1058)$, the level of injury involved the cervical spinal cord. In this cohort, the most common cause of SCI was land transport crash, which accounted for $47 \%(n=1088)$. Other causes included: sports/recreation $(20 \%, n=450)$, violence/ self harm $(18 \%, n=404)$, falls $(7 \%, n=169)$ and other/ unknown $(8 \%, n=186)$.

Data were obtained from USA Model Spinal Cord Injury Systems and enroled in the National Spinal Cord Injury Database for the period 1973-2003 and the catchment area of USA for children aged 0-15 years [18]. In total, 1130 cases of paediatric SCI were identified. No data was reported on SCDys. Quality ranking of data is Moderate Yellow and Orange Zone data.

In this cohort, the most common cause of SCI was land transport crash, which accounted for $44 \%(n=500)$. Other causes included: sports/recreation $(23 \%, n=265)$, violence/ self harm $(21 \%, n=244)$, falls $(8 \%, n=94)$, and other/ unknown $(3 \%, n=33)$. 
Data were obtained from the Nationwide Emergency Department Sample (contains records of approximately 960 hospital-based emergency departments, comprising $20 \%$ of all hospital-based emergency departments in the USA) for the period 2007-2010 and the catchment area of USA for children were aged 0-17 years [19]. In total, 6132 cases of paediatric SCI were identified. This equates to an incidence rate for SCI of 17.5 cases per million population per year. No data was reported on SCDys. Moderate Yellow and Orange Zone data.

Of 6132 SCI cases, $72.5 \%(n=4,447)$ were male. In $40 \%$ of SCI cases $(n=2483)$, the level of injury involved the cervical spinal cord. In this cohort, the most common cause of SCI was land transport, which accounted for $34 \%$ ( $n=2111)$. Other causes included: violence/self harm (23\%, $n=1437)$, falls $(19 \%, n=1164)$, sports/recreation $(6 \%, n=391)$ and other/unknown $(17 \%, n=1029)$.

Data for SCI were obtained from the USA Model Spinal Cord Injury Systems or the Shriners Hospital for Children SCI unit for the period 1973-2004 for children aged less than 16 years. These children were not ventilator dependent and had survived for more than 2 years after injury [20]. No data was reported on SCDys. Quality ranking of data is Moderate Yellow and Orange Zone data.

A total of 25,340 persons contributed 274,020 personyears of data, with 3844 deaths, over the study period. Children who were less than 16 years of age at time of SCI had a $31 \%$ increase in the annual odds of dying compared with persons injured at older ages. The increased risk did not vary significantly by current age, sex, race, injury severity, or decade of injury.

Data for SCDys due to tumour were obtained from the USA central cancer registries in the National Program of Cancer Registries and Surveillance, Epidemiology, and End Results (SEER) programs databases for the period 2004-2007 and the catchment area of over 99\% of the US for children aged 0-19 years [28]. No data was reported for other causes of SCDys or SCI. Quality ranking of data is High and Yellow Zone data.

Of the 869 paediatric SCDys cases due to tumour, $51 \%$ $(n=437)$ were malignant and $49 \%(n=432)$ were nonmalignant primary spinal tumour. This equates to an incidence rate for SCDys of 2.7 cases per million population per year, and were generally equally distributed by sex.

Data were obtained from the population-based cancer registries of the SEER program for the period 1973-2007 and the catchment area of about $26 \%$ of the US population for children were aged 0-18 years [27]. In total, 42 cases of paediatric SCDys due to primary malignant astrocytomas of the spinal cord (PMASC) were identified. Quality ranking of data is High and Yellow Zone data.

When compared with adult cases, the paediatric cohort had survival rates lower than the adult cohort (9-month median survival versus 16-months in the adults) Paediatric mortality at one year for SCDys due to PMASC was $31 \%$ $(n=13)$ and the mortality from one year to 10 years was an additional $12 \%(n=5)$. Sex also had an effect on survival with females having a poorer prognosis.

Data were obtained from the population-based cancer registries of the Surveillance, Epidemiology and End Results (SEER 17) program for the period 1973-2008 and the catchment area of about $26 \%$ of the US population for children aged 0-18 years [26]. In total, 48 cases of paediatric SCDys due to primary high grade spinal cord astrocytomas were identified. Quality ranking of data is Moderate and Yellow Zone data.

Of 48 SCDys cases, 50\% $(n=24 / 48)$ were male. Paediatric mortality at one year for SCDys due to primary paediatric high grade spinal cord astrocytomas was $65 \%(n=31)$ and the mortality from one year to 10 years was an additional $21 \%$ $(n=10)$.

\section{Discussion}

Overall, there were relatively few paediatric SCD publications identified in our literature search compared with adult SCI [2] and SCDys [3]. There were more SCI reports than SCDys, and better data quality from High Income than Medium and Low Income countries. The incidence of SCI was generally higher than that reported for SCDys. The most common causes of SCI were land transport, falls and sport. The most common causes of SCDys were neoplasm and inflammatory. There were very few reports on prevalence, which was higher for SCI, and mortality, which was higher for SCDys.

As with previous global mapping findings [2, 3], there was poorer quality epidemiological data for SCDys than there was for SCI. One reason for this most likely arises from the exclusion of SCDys by many spinal registries. Furthermore, in adults, many traditional Spinal Cord Injury Units or Rehabilitation Centres have been established with a SCI focus and often exclude people with SCDys, or subgroups with these disorders [32]. It is uncertain the degree to which this occurs in the paediatric setting. There are many differences in the results between and within countries, and between SCI and SCDys. Given the relatively few studies conducted in this field, and the paucity of high quality studies and the differing methodologies, it is not appropriate, we feel, to go into a detailed discussion about possible explanations for these differences. More, better quality studies are required before this would be appropriate.

A major strength of this paper is that it is the most comprehensive review of the epidemiology of paediatric SCD published to date. This paper also completes the series of ISCoS Prevention Committee Global Mapping of SCD 
publications. It is planned that these will be updated as new publications are identified.

A major limitation of this project is that there is a scarcity of quality research in the field of paediatric SCI and SCDys epidemiology-highlighted by the absence of Green and Yellow information zones in many regions (Figs. 1 and 2). Overall, the number of included publications was very low considering the global nature of this paper. No country had multiple confirmatory high quality studies covering the whole population. The scope and depth of information contained in most of the studies is very limited. In addition, there was no internationally accepted classification of SCDys [7] available at the time that many of the studies included in this review were conducted. This limited the ability to report and compare the aetiology of SCDys across many countries and Global Regions.

As highlighted in the previously published global mapping of SCDys, there are problems with some studies mixing chronic SCDys (for example, neural tube defects) with new onset SCDys patients when describing the study sample [7]. Another major limitation was that studies used different age cut-offs for inclusion in the paediatric category. We initially planned to use an age cut-off for inclusion of less than 15 years of age to avoid an overlap between the paediatric global maps and the earlier published adult global maps. However, with this inaugural iteration of the paediatric global maps, we have decided to include a selection of papers where it was not possible to clearly parse out patients under the age of 15 , or some regions would have been completely without any paediatric data (these papers are highlighted in the tables).

There are a number of implications from our findings for future research. It is anticipated that future researchers will be more likely to follow the ISCoS guidelines on age grouping and the classification of SCI [5] and SCDys [7]. This will help improve the quality of future studies. In addition, it is necessary to encourage the establishment of population-based regional or national data collection projects to allow more representative data. As a result of these improvements, future research will then be better placed to target prevention programs to reduce paediatric SCD incidence.

\section{Conclusions}

There is a scarcity of quality studies regarding the epidemiology, aetiology and survival following paediatric causes of SCI and SCDys. Recent ISCoS frameworks provide better guidance for classification of SCDys and age group cut-off levels in future studies.

Acknowledgements We would like to thank Erin Gardner and Izzy Chapman for their assistance with the initial work on the literature search, and Christiani Tjandra, Alysia Wong and Paul Peng for their help in retrieving source articles. Dr. Vanessa Noonan [30] and Ms Jane Galvin [29] are thanked for providing additional clarifying information that enabled us to utilise their manuscripts in this project.

Funding: No funding was provided for this research

Author contributions PWN conceived the project, designed and carried out the literature search. He helped review potential publications for inclusion and wrote the initial draft of the manuscript and was responsible for coordinating the subsequent revisions. BBL helped refine the project methodology, reviewed potential publications for inclusion, graded the quality of included publications and assisted in the manuscript revisions. $\mathrm{RC}$ helped refine the project methodology, reviewed and graded the quality of included publications and assisted in the manuscript production and revisions. $\mathrm{He}$ coordinated and extracted data from the included publications into a purposely built database for production of tables and mapping data, managed the referencing and produced the maps. LV, AS and MCW helped refine the project methodology, reviewed potential publications for inclusion and assisted in the manuscript revisions.

Online supplement Appendix 1 shows the search strategy used to identify articles on paediatric SCD from any cause, focusing on the aetiology, incidence, prevalence and mortality. Appendix 2 shows a flow chart with the results of the literature search.

\section{Compliance with ethical standards}

Conflict of interest The authors declare that they have no conflict of interest.

\section{References}

1. New PW, Delafosse V. What to call spinal cord damage not due to trauma? Implications for literature searching. J Spinal Cord Med. 2012;35:89-95.

2. Lee B, Cripps R, Fitzharris M, Wing P. The global map for traumatic spinal cord injury epidemiology: update 2011, global incidence rate. Spinal Cord. 2014;52:110-6.

3. New PW, Cripps R, Lee BB. A global map for non-traumatic spinal cord injury epidemiology: towards a living data repository. Spinal Cord. 2014;52:97-109.

4. Smith E, Finn S, Fitzpatrick P. Epidemiology of Pediatric Traumatic and Acquired Nontraumatic Spinal Cord Injury in Ireland. Top Spinal Cord Inj Rehabil. 2017;23:279-84.

5. Biering-Sorensen F, DeVivo M, Charlifue S, Chen Y, New P, Noonan $\mathrm{V}$, et al. International spinal cord injury core data set (version 2.0)—including standardization of reporting. Spinal Cord. 2017;55:759-64.

6. Harvard University, Institute for Health Metrics and Evaluation at the University of Wasington, Johns Hopkins University, University of Queensland, World Health Organization (WHO). Global burden of disease operations manual, final draft. 2009. Available at https://citeseerx.ist.psu.edu/viewdoc/download?doi= 10.1.1.177.9813\&rep $=$ rep1\&type $=$ pdf Accessed 29th October 2018.

7. New P, Marshall R. International spinal cord injury data sets for non-traumatic spinal cord injury. Spinal Cord. 2014;52:123-32.

8. New PW, Baxter D, Farry A, Noonan V. Estimating the Incidence and prevalence of traumatic spinal cord injury (TSCI) in Australia. Arch Phys Med Rehabil. 2015;96:76-83. 
9. Majdan M, Brazinova A, Mauritz W. Epidemiology of traumatic spinal cord injuries in Austria 2002-2012. Eur Spine J. 2016;25:62-73.

10. Pickett W, Simpson K, Walker J, Brison RJ. Traumatic spinal cord injury in Ontario, Canada. J Trauma Inj Infect Crit Care. 2003;55:1070-6.

11. Knutsdottir S, Thorisdottir $\mathrm{H}$, Sigvaldason $\mathrm{K}$, Jonsson $\mathrm{H} \mathrm{Jr}$, Bjornsson A, et al. Epidemiology of traumatic spinal cord injuries in Iceland from 1975 to 2009. Spinal Cord. 2012;50(2):123-6.

12. Dixon GS, Danesh JN, Caradoc-Davies TH. Epidemiology of spinal cord injury in New Zealand. Neuroepidemiology. 1993; 12:88-95.

13. Hagen EM, Eide GE, Elgen I. Traumatic spinal cord injury among children and adolescents; a cohort study in western Norway. Spinal Cord. 2011;49:981-5.

14. Al-Habib A, Alaqeel A, Marwa I, Almohammadi M, Al Shalaan $\mathrm{H}$, AlEissa S, et al. Causes and patterns of spine trauma in children and adolescents in Saudi Arabia: implications for injury prevention. Ann Saudi Med. 2014;34:31-7.

15. Augutis M, Levi R. Pediatric spinal cord injury in Sweden: incidence, etiology and outcome. Spinal Cord. 2003;41:328-36.

16. Martin B, Dykes E, Lecky F. Patterns and risks in spinal trauma. Arch Dis Child. 2004;89:860-5.

17. DeVivo MJ, Vogel LC. Epidemiology of spinal cord injury in children and adolescents. Spinal Cord Med. 2004;27:S4-10.

18. Jackson AB, Dijkers M, DeVivo MJ, Poczatek RB. A demographic profile of new traumatic spinal cord injuries: change and stability over 30 years. Arch Phys Med Rehabil. 2004;85 (November):1740-8.

19. Selvarajah S, Schneider EB, Becker D, Sadowsky CL, Haider A, Hammond E. The epidemiology of childhood and adolescent traumatic spinal cord injury in the United States: 2007-2010. J Neurotrauma. 2014;31:1548-60.

20. Shavelle RM, DeVivo MJ, Paculdo DR, Vogel LC, Strauss DJ. Long-term survival after childhood spinal cord injury. J Spinal Cord Med. 2007;30(Supplement 1):S48-54.

21. Chien LC, Wu JC, Chen YC, Liu L, Huang WC, Chen TJ, et al. Age, sex, and socio-economic status affect the incidence of pediatric spinal cord injury: an 11-year national cohort study. PLoS ONE. 2012;7:e39264.

22. New PW, Farry A, Baxter D, Noonan VK. Prevalence of nontraumatic spinal cord injuryin Victoria, Australia. Spinal Cord. 2013;51:99-102.

23. van den Berg ME, Castellote JM, Mahillo-Fernandez I, de PedroCuesta J. Incidence of nontraumatic spinal cord injury: a Spanish cohort study (1972-2008). Arch Phys Med Rehabil. 2012;93:325-31.

24. Absoud M, Lim MJ, Chong WK, De Goede CG, Foster K, Gunny $\mathrm{R}$, et al. Paediatric acquired demyelinating syndromes: Incidence, clinical and magnetic resonance imaging features. Mult Scler. 2013;19:76-86.

25. Sharpe AN, Forsyth R. Acute paediatric paraplegia: a case series review. Eur J Paediatr Neurol. 2013;17:620-4.

26. Lam S, Lin Y, Melkonian S. Analysis of risk factors and survival in pediatric high-grade spinal cord astrocytoma: a populationbased study. Pediatr Neurosurg. 2012;48:299-305.

27. Adams H, Avendano J, Raza SM, Gokaslan ZL, Jallo GI, Quinones-Hinojosa A. Prognostic factors and survival in primary malignant astrocytomas of the spinal cord: a population-based analysis from 1973 to 2007. Spine. 2012;37:E727-35.

28. Duong LM, McCarthy BJ, McLendon RE, Dolecek TA, Kruchko C, Douglas LL, et al. Descriptive epidemiology of malignant and nonmalignant primary spinal cord, spinal meninges, and cauda equina tumors, United States, 2004-2007. Cancer . 2012;118: 4220-7.

29. Galvin J, Scheinberg A, New PW. A retrospective case series of pediatric spinal cord injury and disease in Victoria, Australia. Spine. 2013;38:E878-82.

30. Noonan VK, Fingas M, Farry A, Baxter D, Singh A, Fehlings MG, et al. Incidence and prevalence of spinal cord injury in Canada: a national perspective. Neuroepidemiology. 2012;38:219-26.

31. Lee JH, Sung IY, Kang JY, Park SR. Characteristics of pediatriconset spinal cord injury. Pediatr Int. 2009;51:254-7.

32. New PW. Non-traumatic spinal cord injury: what is the ideal setting for rehabilitation? Aust Health Rev. 2006;30:353-61.

\section{Affiliations}

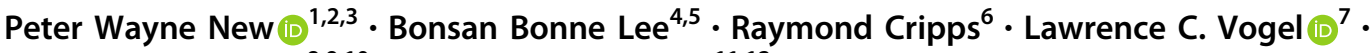 Adam Scheinberg (10) ${ }^{8,9,10} \cdot$ Mary-Clare Waugh (10) ${ }^{11,12}$}

1 Spinal Rehabilitation Service, Caulfield Hospital, Alfred Health, Melbourne, Victoria, Australia

2 Epworth-Monash Rehabilitation Medicine Unit, Monash University, Melbourne, Victoria, Australia

3 Department of Epidemiology and Preventive Medicine, School of Public Health \& Preventive Medicine, Monash University, Melbourne, Victoria, Australia

4 Prince of Wales Spinal Medicine Department, Prince of Wales Hospital, Sydney, NSW, Australia

5 Neuroscience Research Australia (NEURA) Sydney, Sydney, NSW, Australia

6 Flinders University, College of Medicine and Public Health, Research Centre for Injury Studies, Adelaide, South Australia, Australia
7 Shriners Hospitals for Children, Chicago, IL, USA

8 Royal Children's Hospital, Paediatric Rehabilitation Unit, Melbourne, Victoria, Australia

9 Murdoch Childrens Research Institute, Victoria, Australia

10 Department of Paediatrics, University of Melbourne, Victoria, Australia

11 Kids Rehab, The Children's Hospital at Westmead, Westmead, NSW, Australia

12 Department of Paediatrics, University of Sydney, Sydney, NSW, Australia 